\title{
Differential genome-wide profiling of alternative polyadenylation sites in nasopharyngeal carcinoma by high- throughput sequencing
}

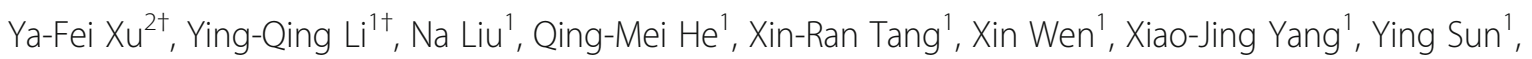
Jun $\mathrm{Ma}^{1 *}$ and Ling-Long Tang ${ }^{1^{*}}$

\begin{abstract}
Background: Alternative polyadenylation (APA) is a widespread phenomenon in the posttranscriptional regulation of gene expression that generates mRNAs with alternative 3'-untranslated regions (3'UTRs). APA contributes to the pathogenesis of various diseases, including cancer. However, the potential role of APA in the development of nasopharyngeal carcinoma (NPC) remains largely unknown.

Methods: A strategy of sequencing APA sites (SAPAS) based on second-generation sequencing technology was carried out to explore the global patterns of APA sites and identify genes with tandem 3'UTRs in samples from 6 NPC and 6 normal nasopharyngeal epithelial tissue (NNET). Sequencing results were then validated using quantitative RT-PCR in a larger cohort of 16 NPC and 16 NNET samples.

Results: The sequencing data showed that the use of tandem APA sites was prevalent in NPC, and numerous genes with APA-switching events were discovered. In total, we identified 195 genes with significant differences in the tandem 3'UTR length between NPC and NNET; including 119 genes switching to distal poly (A) sites and 76 genes switching to proximal poly (A) sites. Several gene ontology (GO) terms were enriched in the list of genes with switched APA sites, including regulation of cell migration, macromolecule catabolic process, protein catabolic process, proteolysis, small conjugating protein ligase activity, and ubiquitin-protein ligase activity.

Conclusions: APA site-switching events are prevalent in NPC. APA-mediated regulation of gene expression may play an important role in the development of NPC, and more detailed studies targeting genes with APA-switching events may contribute to the development of novel future therapeutic strategies for NPC.
\end{abstract}

Keywords: Alternative polyadenylation, Genome-wide profiling, High-throughput sequencing, Nasopharyngeal carcinoma

\section{Background}

Nasopharyngeal carcinoma (NPC) is an epithelial malignancy of the head and neck with a highly unbalanced ethnic and geographic distribution. It occurs mainly in Southern China and Southeast Asia, where the incidence

\footnotetext{
* Correspondence: majun2@mail.sysu.edu.cn; tangll@sysucc.org.cn ${ }^{\dagger}$ Ya-Fei Xu and Ying-Qing Li contributed equally to this work.

${ }^{1}$ Sun Yat-sen University Cancer Center; State Key Laboratory of Oncology in South China; Collaborative Innovation Center for Cancer Medicine; Guangdong Key Laboratory of Nasopharyngeal Carcinoma Diagnosis and Therapy, Guangzhou 510060, People's Republic of China

Full list of author information is available at the end of the article
}

can be as high as 20 to 50 cases per 100,000 person-years [1-3]. Although the 5-year overall survival rate is approximately $60 \%[4]$ and has increased with the advent of intensity-modulated radiation therapy [5], the prognosis of NPC is still very poor due to recurrence or/and distant metastasis [6]. The etiology of NPC is multi-factorial; including genetic components, Epstein-Barr virus (EBV) infection, environmental factors, and interactions between these factors [7-9]. Among these factors, genetic components play a vital role in the development of NPC and various genes related to NPC have been identified [10-13]. However,

(c) The Author(s). 2018 Open Access This article is distributed under the terms of the Creative Commons Attribution 4.0 International License (http://creativecommons.org/licenses/by/4.0/), which permits unrestricted use, distribution, and 
genomic abnormalities in NPC tumorigenesis remain largely unidentified. It is particularly important that the genomic foundations of NPC are clearly defined to identify genes contributing to the initiation and progression of NPC, and further guide the development of novel therapeutic strategies for NPC patients.

Posttranscriptional regulation at the mRNA level is generally mediated by miRNAs and RNA-binding proteins which recognize and bind to elements within the 3 '-untranslated region (3'UTR). Dysregulation of this can perturb gene expression and contribute to the pathogenesis of various diseases, including several types of cancer [14, 15]. The eukaryotic transcriptome is particularly complicated due to its use of alternative polyadenylation (APA) sites, which can generate diverse mRNA isoforms from a single gene that differ either in their coding sequence, their 3'UTRs, or both [16]. APA is a widespread phenomenon, with more than half of the genes in humans and over $30 \%$ in mice having numerous APA sites [17]. Recent studies have shown that APA-switching events may occur in a tissue- or disease-specific manner [18]. The mRNA transcripts in placenta, ovaries and blood are characterized by preferential usage of proximal poly(A) sites (generating isoforms with shorter 3'UTRs), whereas in nervous system and brain, they tend to use distal poly(A) sites (generating isoforms with longer 3'UTRs) [19].

Tandem 3'UTRs can also affect mRNA stability, translation efficiency, and subcellular localization by causing loss of regulatory elements, especially miRNA binding sites in the 3'UTR [20-22]. APA-switching events can influence a number of critical biological processes, including embryonic development and cell differentiation [23-25], cell proliferation [26, 27], immune response [22], neuron activation [28, 29], and tumorigenesis [27, $30-32$ ]. It has been shown that activated $\mathrm{T}$ lymphocytes [22] and cancer cells [27] generally use shorter 3'UTRs, and that shortening of tandem 3'UTRs is associated with cell proliferation [22] while the lengthening of tandem 3'UTRs has been observed during cell differentiation $[24,33]$. study by Mayr et al. showed that shortening of tandem 3'UTRs was preferentially used by several oncogenes in cancer cell lines [27], suggesting APA-mediated gene expression may play an important role in cancer development. Recent studies also reported that APA-switching events occurred in various types of cancer, including breast cancer [30], colorectal carcinoma [32], glioblastoma [34], and gastric cancer [35]. Nevertheless, whether APA-switching events are integral to the development and progression of NPC remains unknown.

In this study, for the first time, we performed genome-wide profiling of APA sites, with a sequencing APA sites (SAPAS) technology using second-generation sequencing, in NPC and normal nasopharyngeal epithelial tissues (NNET) to identify genes with 3'UTR switching that participate in NPC development. Gene Ontology (GO) and pathway analysis were performed to better understand the function of genes with 3'UTR switching. Subsequently, we validated our sequencing results by quantitative RT-PCR in a larger sample size. Our research provides a novel insight into the tumorigenesis of NPC.

\section{Methods}

Clinical specimens

Sixteen NPC fresh tissue samples were collected at the Department of Radiation Oncology of Sun Yat-sen University Cancer Center (Guangzhou, China) between 16 Jan 2010 and 25 Feb 2013. All samples were reassessed by two pathologists and the percentage of tumor cells was $70 \%$ or more in all samples. None of the patients had received radiotherapy or chemotherapy before biopsy sampling. 16 NNET tissue samples were collected from outpatients, showed no evidence of cancer, and the tissue samples showed normal histology. This study was approved by the Human Ethics Approval Committee at Sun Yat-sen University Cancer Center. Written informed consent was obtained from each study subject.

\section{RNA extraction}

Total RNA was extracted using TRIzol reagent (Life Technologies, Grand Island, NY, USA) according to the manufacturer's instructions. Genomic DNA was removed from total RNA by TURBO DNase (Ambion, Austin, TX, USA). The quantity of RNA was determined using a NanoDrop2000 spectrophotometer (Thermo Scientific, Wilmington, DE, USA) and RNA purity was assessed by the ratio of absorbance at 260 and $280 \mathrm{~nm}$. RNA quality was assessed using electrophoresis in a $1.5 \%$ agarose gel stained with ethidium bromide.

\section{Construction of the $3^{\prime} U T R$ library}

The SAPAS sequencing libraries were constructed as described previously $[30,36]$. In brief, total RNA was randomly fragmented by heating. The first strand cDNA was generated by a template-switch reverse transcription (RT) reaction, in which contains an anchored oligo $\mathrm{d}(\mathrm{T})$ primer, a 5' template switching adaptor, and Super-Script II kits (Invitrogen Life Technologies, Karlsruhe, Germany). Then, ds-cDNA was amplified by PCR using known primers tagged with Illumina adaptors. The sequences of all primers were consistent with previous studies [30, 37]. PAGE gel-excision was carried out to select the PCR products with the fragments of 300-500 bp with a QIAquick Gel Extraction Kit (Qiagen, Valencia, CA). The final pooled fragments were sequenced from their 3'end on the Illumina HiSeq 2000 system in dark cycle mode. Finally 58 bp reads were generated. 


\section{Illumina reads filtering and mapping}

Reads were filtered and trimmed with in-house Perlscripts, and the trimmed reads were aligned to the human genome (hg19) with Bowtie. Internal priming filtering was performed by analyzing the genomic sequences located 1 to 20 bases downstream of poly(A) cleavage sites, containing the sequence motifs 5'-AAAAAAAA-3', 5'-GAAAA +GAAA+G-3' ("+" means "or more"), or more than 12 "A"s. Sites with only one read were removed.

\section{Poly(A) sites identification and annotation}

Cleavage sites were clustered into poly(A) sites as described previously $[17,30]$. Briefly, the reads of which 3 ' ends located within $24 \mathrm{nt}$ from each other and which aligned to the same strand of a chromosome were clustered. Then, cleavage clusters with two or more reads were assigned as poly(A) sites. Next, poly(A) sites were grouped into three types according to the known poly(A) sites in the UCSC transcript ends database [38] and Tian's database [17]: 1) UCSC known genes, where the poly(A) site was located within $24 \mathrm{nt}$ from the $3^{\prime}$ transcription end of a UCSC gene; 2) Tian poly(A) DB, where the poly(A) site was located within 24 nt from a poly(A) site in Tian's poly(A) DB; 3) Putative novel poly(A) sites, containing the following six attributes: 3'UTR; located $<=1 \mathrm{~kb}$ nt downstream of a UCSC gene; CDS; intron; intergenic; and noncoding gene. Meanwhile, poly(A) sites with reads that overlapped Ensemble-annotated 3'UTR(s) were defined as tandem poly(A) sites. The expression of mRNAs were accumulated by reads of all poly(A) sites, and then the differential expressed genes were identified by edgeR [39]. The raw sequencing data can be accessed from the NCBI Bioproject (http://www.ncbi.nlm.nih.gov/bioproject) under accession no. PRJNA299088.

\section{3'UTR switching analysis between NPC and NNET and functional annotation}

3'UTR switching of each gene between 6 NPC and 6 NNET tissues was detected by a pair-wise case-control analysis, which sequentially compared NPC samples to NNET samples to identify genes with 3'UTR switching in each pair. They were then filtered using the Benjamini-Hochberg false discovery rate (FDR), whereby those estimated to be below 0.01 (using $\mathrm{R}$ software, version 2.15) were kept. In total, genes with 3'UTR switching that occurrences in more than 10 pairs were defined as genes with tandem 3'UTR between NPC and NNET. Functional annotation analysis of these genes was performed using DAVID Bio-informatics Resources (http:// david.abcc.ncifcrf.gov/) [40]. The miRNA targets involved in APA-switching events were identified by TargetScan database [41].

\section{Validation of quantitative RT-PCR analysis}

To validate the sequencing data, quantitative RT-PCR was carried out in 16 NPC and 16 NNET tissues for eight genes (JAG1, IRF1, EGLN1, TIMP3, WDR5, $S M A D 3, F N D C 3 B$, and XRCC5) with extreme 3'UTR length differences between NPC and NNET. Based on SAPAS data, the poly(A) sites of each gene were divided into two supersites: "proximal sites" and "distal sites". Two gene-specific primer sets were specifically designed for "proximal sites" and "distal sites", as described previously [30]. Primer sequences are listed in Additional file 1. The quantitative RT-PCR was performed on the CFX96TouchTM sequence detection system (Bio-Rad, Hercules, CA, USA) using Platinum SYBR Green qPCR SuperMix-UDG reagents (Invitrogen) according to the manufacturer's instructions. GAPDH was used as a control for normalization. For each gene, the relative expression ratio of the proximal site to the distal site was calculated using the $2^{-\Delta \Delta C T}$ method [42].

\section{Statistical analysis}

SPSS 16.0 software was used for statistical analysis. All of the data were presented as the mean \pm SD. The Student's t-test was used to determine whether significant differences existed in the usage of poly(A) sites of genes between two groups, and two-tailed $P<0.05$ was considered significant.

\section{Results}

\section{Global deep sequencing of 3 ' ends of mRNA}

We used the SAPAS strategy to profile APA sites of 6 NPC and 6 NNET tissues. In total, 20.99 to 26.97 million raw reads with lengths of $58 \mathrm{bp}$ were obtained from Illumina sequencing. A statistical summary of the data is shown in Table 1. Approximately 20.83 to 26.64 million reads harbored the modified anchor oligo $\mathrm{d}(\mathrm{T})$, of which 13.02 to 15.71 million reads uniquely mapped to the human nucleus genome (hg19) [43]. Furthermore, 9.83 to 12.68 million reads that could be used directly to infer transcript cleavage sites were obtained after filtering the reads with internal priming. In total, 21,095 UCSC canonical genes were sequenced by at least one read, which accounted for $27.2 \%$ of all canonical genes.

\section{A comprehensive inventory of $\operatorname{poly}(A)$ sites}

As shown in Fig. 1a, the majority of filtered reads $(82.17 \%)$ were mapped to known poly(A) sites listed in the UCSC transcripts ends database [38] and Tian's database [17]. An additional $5.49 \%$ and $1.29 \%$ of reads were mapped to the 3 'UTR and $1 \mathrm{~kb}$ region downstream from the UCSC canonical genes, respectively. The distribution of the number of all reads is shown in Fig. 1b. As a result of the heterogeneity of cleavage sites at poly(A) sites, we took the cleavage clusters with more than one 
Table 1 Summary of the SAPAS data from Illumina HiSeq 2000 sequencing

\begin{tabular}{|c|c|c|c|c|c|c|c|c|c|c|c|c|}
\hline & \multicolumn{6}{|l|}{ NPC } & \multicolumn{6}{|l|}{ NNET } \\
\hline & NPC1 & NPC2 & NPC3 & NPC4 & NPC5 & NPC6 & NNET1 & NNET2 & NNET3 & NNET4 & NNET5 & NNET6 \\
\hline Raw reads $\left(M^{*}\right)$ & 26.08 & 23.81 & 24.48 & 25.29 & 25.14 & 20.99 & 25.94 & 23.91 & 25.77 & 24.87 & 26.97 & 24.56 \\
\hline Clean reads $(\mathrm{M})$ & 25.90 & 23.54 & 24.06 & 24.94 & 24.94 & 20.83 & 25.77 & 23.59 & 25.33 & 24.70 & 26.64 & 24.42 \\
\hline Mapped to genome(M) & 21.73 & 19.75 & 20.02 & 21.06 & 21.39 & 17.45 & 21.28 & 19.49 & 21.09 & 19.53 & 19.46 & 21.10 \\
\hline Uniquely mapped to genome(M) & 17.02 & 16.42 & 16.11 & 16.82 & 17.29 & 14.33 & 17.45 & 15.87 & 17.75 & 16.17 & 15.72 & 17.48 \\
\hline Mapped to nuclear genome(M) & 13.32 & 14.63 & 13.11 & 13.02 & 14.69 & 13.34 & 15.21 & 14.45 & 15.71 & 14.57 & 13.92 & 14.76 \\
\hline Passed Internal Priming filter(M) & 11.51 & 9.83 & 10.18 & 10.30 & 12.68 & 11.36 & 12.00 & 11.47 & 10.01 & 12.50 & 12.06 & 12.36 \\
\hline Genes sampled by reads $(10 \mathrm{~K})$ & 1.78 & 1.89 & 1.88 & 1.90 & 1.80 & 1.83 & 1.86 & 1.88 & 1.91 & 1.88 & 1.73 & 1.87 \\
\hline Poly(A) sites $(10 \mathrm{~K})$ & 13.70 & 29.99 & 17.36 & 19.04 & 13.82 & 12.50 & 22.75 & 18.14 & 36.03 & 16.28 & 12.45 & 14.90 \\
\hline Known poly(A) sites sampled (10 K) & 2.75 & 2.90 & 2.89 & 2.89 & 2.82 & 2.87 & 2.84 & 2.92 & 2.86 & 2.97 & 2.67 & 2.90 \\
\hline Putative novel poly(A) sites $(10 \mathrm{~K})$ & 10.95 & 27.09 & 14.47 & 16.15 & 11.00 & 9.63 & 19.91 & 15.21 & 33.17 & 13.31 & 9.78 & 12.01 \\
\hline Genes sampled by poly $(\mathrm{A})$ sites $(10 \mathrm{~K})$ & 1.62 & 1.72 & 1.71 & 1.73 & 1.63 & 1.66 & 1.73 & 1.72 & 1.73 & 1.72 & 1.53 & 1.72 \\
\hline
\end{tabular}
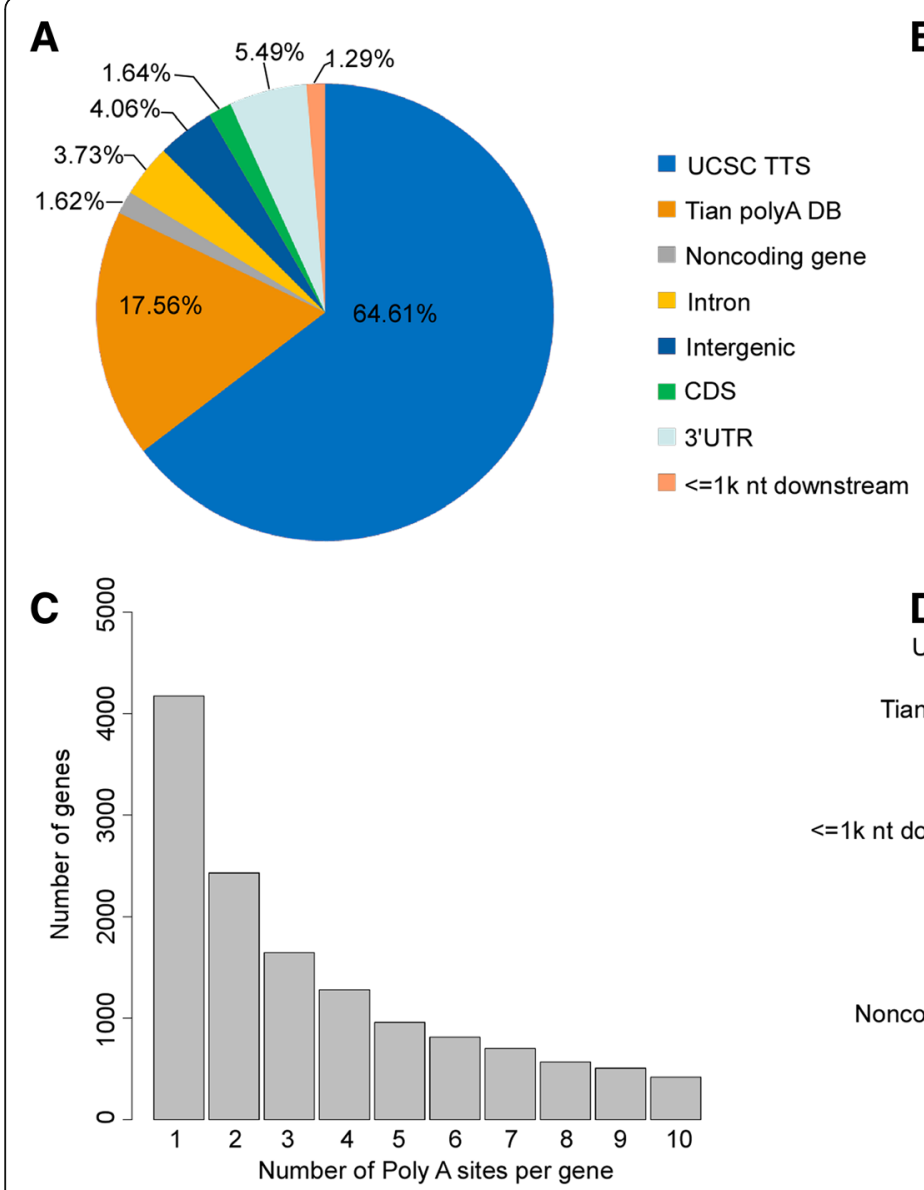
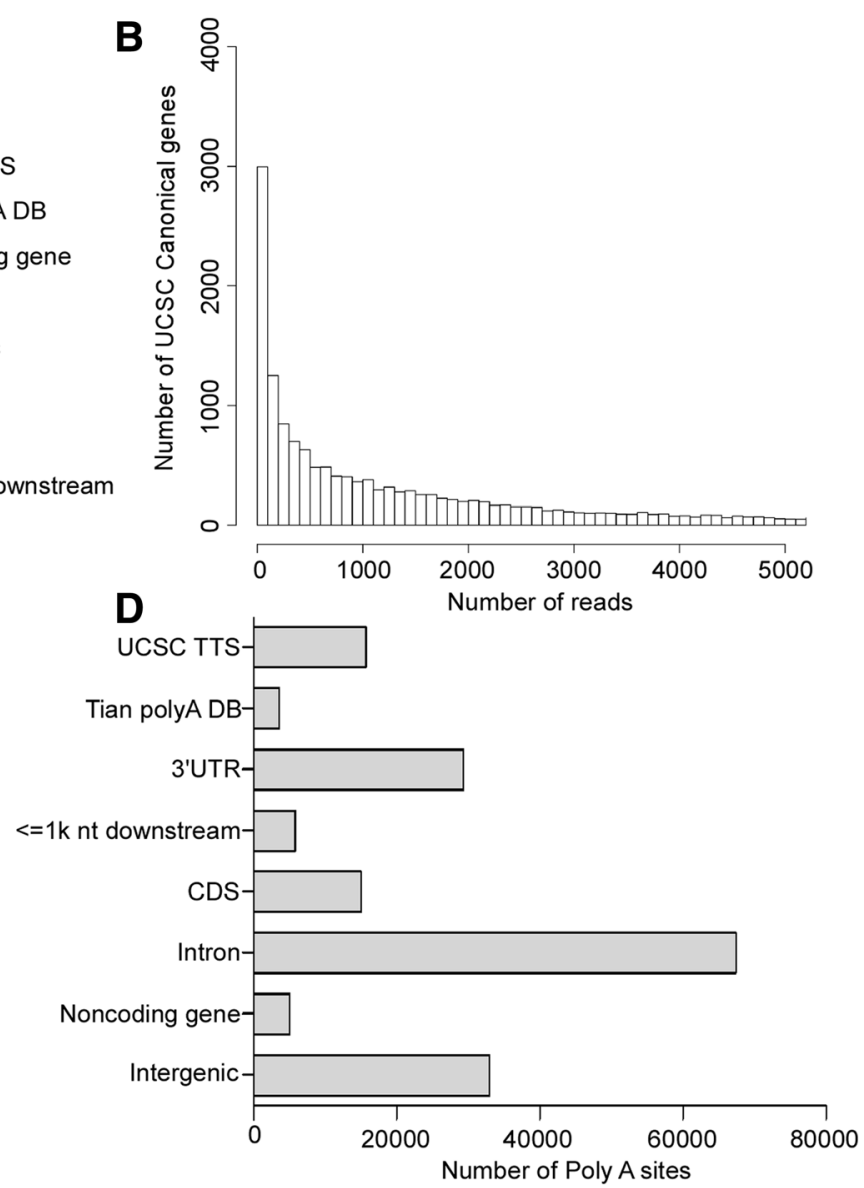

Fig. 1 The characteristics of the SAPAS data. a The genomic locations of reads that specifically aligned to the nuclear genome after internal priming filtering. $\mathbf{b}$ The histogram of the number of reads for UCSC canonical genes. $\mathbf{c}$ The distribution of numbers of poly(A) sites per gene. $\mathbf{d}$ The genomic locations of poly(A) sites for UCSC canonical genes 
read as poly(A) sites. We observed that 13,181 genes had more than one tandem APA site, among which 10,753 genes harbored more than two tandem APA sites (Fig. 1c). In total, 174,931 poly(A) sites were identified from all twelve samples. Only $11.04 \%$ of these sites were found in the UCSC and Tian databases. Another 16.78\% of the poly(A) sites were found in the 3'UTRs, 3.31\% within $1 \mathrm{~kb}$ downstream from the UCSC canonical genes, $18.86 \%$ in intergenic, $38.57 \%$ in intron, $2.87 \%$ in noncoding genes, and $8.56 \%$ in CDS from the UCSC canonical genes (Fig. 1d).

\section{Differential usage of poly(A) between NPC and NNET}

Previous studies have demonstrated that most cancer cells [27] and cancer tissues [31, 32] tend to use shortened 3'UTRs. In contrast to these results for primary cancers, however, one study reported that a greater number of genes with lengthened 3'UTRs existed in a metastasis cell line [30]. In our study, the 3'UTR length of each gene was calculated according to the distance between the poly(A) sites and the stop codon. Subsequently, a pair-wise case-control analysis between NPC and NNET tissues was performed, and a total of 36 pairs were obtained. 3'UTR switching for each gene within each pair was detected using a test of linear trend alternative to independence [44]. A positive Pearson correlation coefficient $(r)$ indicates that NPC tissue contains more lengthened tandem 3'UTRs than NNET, while a negative indicates that NPC tissue harbors more shortened tandem 3'UTRs. The genes with 3'UTR switching between NPC and NNET obtained for each pair are shown in Table 2. We further analyzed the 3'UTR switching frequency for each gene in 36 pairs and found that the frequency of the genes with 3'UTR switching was the highest in at least 10 pairs (Additional file 2). Therefore, we set 10 pairs as the selection criteria for further screening of genes with 3'UTR switching. As a result, we identified 195 genes with significantly different 3'UTR lengths (false discovery rate $[\mathrm{FDR}]<0.01, P<$ 0.01 ), of which 119 genes switched to longer 3'UTRs and 76 genes switched to shorter 3'UTRs in NPC. Details of the 195 genes with significant differences between NPC and NNET are shown in Additional file 3.

\section{Functional annotation analysis of the genes with switched APA sites}

To explore the biological significance of these APA site-switching genes, a functional annotation of the above 195 genes was performed with Database for Annotation, Visualization and Integrated Discovery (DAVID) Bio-informatics Resources, where single-UTR genes were set as the background. The results of Gene Ontology (GO) terms analysis showed that genes with tandem 3'UTRs were mainly enriched in the regulation
Table 2 Genes with APA site switching between NPC and NNET tissues using a pair-wise case-control analysis

\begin{tabular}{|c|c|c|c|}
\hline Pairs & $\begin{array}{l}\text { 3'UTR } \\
\text { shortened } \\
\text { genes }\end{array}$ & $\begin{array}{l}\text { 3'UTR } \\
\text { lengthened } \\
\text { genes }\end{array}$ & Combined \\
\hline NPC1 vs. NNET1 & 104 & 372 & 476 \\
\hline NPC1 vs. NNET2 & 179 & 192 & 371 \\
\hline NPC1 vs. NNET3 & 144 & 247 & 391 \\
\hline NPC1 vs. NNET4 & 58 & 242 & 300 \\
\hline NPC1 vs. NNET5 & 99 & 446 & 545 \\
\hline NPC1 vs. NNET6 & 51 & 123 & 174 \\
\hline NPC2 vs. NNET1 & 55 & 85 & 140 \\
\hline NPC2 vs. NNET2 & 91 & 13 & 104 \\
\hline NPC2 vs. NNET3 & 108 & 38 & 146 \\
\hline NPC2 vs. NNET4 & 100 & 85 & 185 \\
\hline NPC2 vs. NNET5 & 123 & 234 & 357 \\
\hline NPC2 vs. NNET6 & 184 & 106 & 290 \\
\hline NPC3 vs. NNET1 & 63 & 143 & 206 \\
\hline NPC3 vs. NNET2 & 104 & 23 & 127 \\
\hline NPC3 vs. NNET3 & 51 & 26 & 77 \\
\hline NPC3 vs. NNET4 & 79 & 103 & 182 \\
\hline NPC3 vs. NNET5 & 68 & 178 & 246 \\
\hline NPC3 vs. NNET6 & 149 & 111 & 260 \\
\hline NPC4 vs. NNET1 & 62 & 167 & 229 \\
\hline NPC4 vs. NNET2 & 210 & 104 & 314 \\
\hline NPC4 vs. NNET3 & 136 & 115 & 251 \\
\hline NPC4 vs. NNET4 & 158 & 236 & 394 \\
\hline NPC4 vs. NNET5 & 156 & 355 & 511 \\
\hline NPC4 vs. NNET6 & 133 & 125 & 258 \\
\hline NPC5 vs. NNET1 & 158 & 377 & 535 \\
\hline NPC5 vs. NNET2 & 193 & 116 & 309 \\
\hline NPC5 vs. NNET3 & 150 & 153 & 303 \\
\hline NPC5 vs. NNET4 & 55 & 112 & 167 \\
\hline NPC5 vs. NNET5 & 107 & 286 & 393 \\
\hline NPC5 vs. NNET6 & 66 & 82 & 148 \\
\hline NPC6 vs. NNET1 & 102 & 202 & 304 \\
\hline NPC6 vs. NNET2 & 137 & 24 & 161 \\
\hline NPC6 vs. NNET3 & 147 & 99 & 246 \\
\hline NPC6 vs. NNET4 & 53 & 58 & 111 \\
\hline NPC6 vs. NNET5 & 90 & 172 & 262 \\
\hline NPC6 vs. NNET6 & 139 & 76 & 215 \\
\hline
\end{tabular}

of cell migration, macromolecule catabolic process, protein catabolic process, proteolysis, small conjugating protein ligase activity, and ubiquitin-protein ligase activity (Table 3). Additionally, genes with tandem 3'UTRs involved in the ubiquitin-mediated proteolysis pathway $(P=0.022), \quad$ lysosomepathway $\quad(P=0.048), \quad$ colorectal 
Table 3 Enrichment of genes with tandemed 3'UTR isoforms involved in various GO functional categories

\begin{tabular}{|c|c|c|c|}
\hline GO category & & Count & $P$-value \\
\hline GOTERM_BP_FAT & Regulation of cell migration & 9 & 0.0004 \\
\hline GOTERM_BP_FAT & Regulation of locomotion & 9 & 0.001 \\
\hline GOTERM_BP_FAT & Regulation of cell motion & 9 & 0.001 \\
\hline GOTERM_BP_FAT & Macromolecule catabolic process & 19 & 0.0015 \\
\hline GOTERM_BP_FAT & Protein catabolic process & 16 & 0.0024 \\
\hline GOTERM_BP_FAT & Proteolysis & 20 & 0.015 \\
\hline GOTERM_MF_FAT & Small conjugating protein ligase activity & 7 & 0.0052 \\
\hline GOTERM_MF_FAT & Acid-amino acid ligase activity & 7 & 0.013 \\
\hline GOTERM_MF_FAT & Ubiquitin-protein ligase activity & 6 & 0.013 \\
\hline KEGG_PATHWAY & Ubiquitin mediated proteolysis & 6 & 0.022 \\
\hline KEGG_PATHWAY & Lysosome & 5 & 0.048 \\
\hline KEGG_PATHWAY & Colorectal cancer & 5 & 0.017 \\
\hline KEGG_PATHWAY & Renal cell carcinoma & 4 & 0.049 \\
\hline
\end{tabular}

cancer $(P=0.017)$ and renal cell carcinoma pathway $(P=$ 0.049) were enriched (Table 3 ). We also performed GO analysis and pathway enrichment analysis by separating the 195 genes into two groups (switching to distal poly(A) and proximal poly(A)), and results were showed in Additional files 4, 5. Since distant metastasis occurs in $20-30 \%$ of NPC patients and is the major cause of death in NPC [6], it is noteworthy that we identified 9 genes with enriched tandem 3'UTRs that are involved in the regulation of cell migration; namely SMAD3, JAG1, Pikr1, Ptp4a1, Rac1, RRAS2, Spag9, TRIP6, and TRIB1 (Table 4). These results indicate APA site-switching events can influence a number of critical biological processes and may play an important role in the development of NPC.

\section{The effects of APA-site switching events on gene} expression and miRNA targeting sites

To further explore the functional effects of APA-site switching events, we firstly analyzed the relationship between the APA switched sites and the mRNA levels of 195 genes. A total of 275 genes with significant differences in the mRNA levels between NPC and NNET were identified, including 198 genes that were up-regulated and 77 genes that were down-regulated in NPC (Additional file 6). Further analyses revealed that among the 119 genes that switched to longer 3'UTRs in NPC, 4 genes were up-regulated and none was down-regulated; and among the 76 genes that switched to shorter 3'UTRs, only one gene was found to be up-regulated or down-regulated in NPC (Additional file 7).

Table 4 Nine genes enriched in GO terms associated with cell migration

\begin{tabular}{|c|c|c|c|}
\hline UCSC ID & Gene Symbol & Gene Name & $r$-value \\
\hline uc002aqj.2 & $\mathrm{SMAD3}^{\mathrm{a}}$ & SMAD family member 3 & 0.086 \\
\hline uc002wnw.2 & $J A G 1^{a}$ & jagged 1 (Alagille syndrome) & -0.351 \\
\hline uc003jva.2 & Pikr1 & phosphoinositide-3-kinase, regulatory subunit 1 (alpha) & 0.0007 \\
\hline uc003pek.2 & Ptp4a1 & protein tyrosine phosphatase type IVA, member 1 & 0.929 \\
\hline uc003spw.2 & Rac1 & $\begin{array}{l}\text { ras-related C3 botulinum toxin substrate } 1 \\
\text { (rho family, small GTP binding protein Rac1) }\end{array}$ & 0.175 \\
\hline uc009ygq.2 & RRAS2 & $\begin{array}{l}\text { related RAS viral (r-ras) oncogene homolog 2; similar to } \\
\text { related RAS viral (r-ras) oncogene homolog } 2\end{array}$ & -0.564 \\
\hline uc002ita.2 & Spag9 & sperm associated antigen 9 & 0.411 \\
\hline uc003uww.2 & TRIP6 & thyroid hormone receptor interactor 6 & 0.182 \\
\hline uc003yrx.2 & TRIB1 & tribbles homolog 1 (Drosophila) & -0.206 \\
\hline
\end{tabular}

a genes selected for quantitative RT-PCR validation

$r$-value: a positive value of $r$ indicates a longer tandem $3^{\prime} U T R$ in NPC, and vice versa 
These results suggest that the APA-site switching events could not change the mRNA expression levels, and it may affect the translation efficiency and subcellular localization by causing loss of regulatory elements, especially miRNA binding sites in the 3'UTR.

Furthermore, we identified potential miRNA targeting sites between the "proximal polyA sites" and "distal polyA sites" of 195 genes using the publicly available databases TargetScan to explore the effect of APA-switching events on the gain-of or loss-of miRNA targeting sites in their 3'UTR. The results showed that for the 119 genes switched to longer 3'UTRs in NPC, totally 706 miRNA targeting sites were gained and for the 76 genes switched to shorter 3'UTRs in NPC, totally 325 miRNA targeting sites were lost (Additional file 8). These result suggests that APA-switching events can result in the gain or loss of miRNA binding sites in the 3'UTR in the development of NPC.

\section{Real-time RT-PCR validation of selected APA switched sites}

To further validate the SAPAS sequencing data, we randomly selected eight genes (JAG1, IRF1, EGLN1, TIMP3, WDR5, SMAD3, FNDC3B, and XRCC5) with switched APA sites for quantitative RT-PCR validation in 16 NPC and 16 NNET samples. Results of six genes were similar to the sequencing data (Fig. 2). JAG1, IRF1, EGLN1, WDR5, and SMAD3 tended to use lengthened 3'UTR transcripts, whereas $F N D C 3 B$ tended to use shortened 3'UTR transcripts $(P<0.05)$. Consistent with the sequencing data, there appeared to be a higher tendency for lengthened 3'UTR transcripts from the genes TIMP3 and XRCC5 to be present in NPC than NNET, however no statistical differences were observed. These tendencies suggest that APA-switching events were more prevalent in NPC tissues.

\section{Discussion}

APA allows a single gene to encode multiple mRNA transcripts by changing the mRNA 3'UTR length and plays an important role in various physiological and pathological processes, including tumorigenesis $[27,30,45,46]$. In this study, we performed genome-wide profiling of tandem APA sites in NPC and NNET tissues using SAPAS based on second-generation sequencing technology. In total, we identified 195 genes whose tandem 3'UTR length differed significantly between NPC and NNET, including 119 genes switching to distal poly(A) sites and 76 genes switching to proximal poly(A) sites. Several gene ontology (GO) terms were enriched in the list of genes with switched APA sites, including regulation of cell migration, macromolecule catabolic process, protein catabolic process, proteolysis, small conjugating protein ligase activity, and ubiquitin-protein ligase activity. The sequencing results were further validated using quantitative RT-PCR.

APA, which leads to alterations in 3'UTR length and content of genes, shows dynamic characteristics in a variety of tumors. A previous analysis of 27 cancer cell lines derived from sarcomas and breast, lung and colon cancers showed that tumor cells generally use the shorter 3'UTRs [27]. Similarly, Lin et al. found that shorter 3'UTRs isoforms were preferentially upregulated in breast, colon, kidney, liver and lung cancer tissues [31]. Likewise, Morriset al. demonstrated that more genes tended to use shorter 3'UTRs isoforms in colorectal carcinoma compared to colorectal adenoma and normal colon mucosa [32]. However, a recent discovery showed that longer 3'UTRs isoforms were significantly upregulated in MB231, a human breast cancer line that is estrogen independent and highly invasive [30]. Nordlund et al. demonstrated that more genes preferentially used longer 3'UTRs in acute lymphoblastic leukemia [47]. These findings suggest that APA-switching events are more complicated and may occur in a tissue- or disease-specific manner. In this study, we found that 119 genes tended to use longer 3'UTRs and 76 genes tended to use shorter 3'UTRs in NPC, which is the first genome-wide research to investigate APA in NPC.

Furthermore, 3'UTRs harbor several cis-elements, such as U-rich elements (USE), poly A signals (PAS), ARE (AU-rich elements), cytoplasmic polyadenylation elements, miRNA target sites and other unknown elements [46]. Therefore, APA-induced alterations in 3'UTR length and content may result in loss or gain of these regulatory motifs, resulting in series of changes in cell biological function by affecting mRNA stability, transcript export and translation efficiency [48]. Recent studies have revealed that shorter 3'UTRs could lead to greater mRNA stability and increased protein output, and were also associated with elevated cell proliferation rates or transformation [27]. In this study, we identified 195 genes with tandem 3'UTRs isoforms, which were involved in various cellular biological functions, including regulation of cell migration, macromolecule catabolic processes, protein catabolic processes, proteolysis, small conjugating protein ligase activity, and ubiquitin-protein ligase activity. This result is not unexpected because it has been confirmed that metastasis occurs in $20-30 \%$ of NPC patients and is the major cause of death in NPC [6]. It is important to note that our study effectively identified genes that were involved in NPC tumorigenesis.

To date, several possible mechanisms have been reported to influence the regulation of APA-switching events. Previous studies have demonstrated that the usage of APA could be influenced by the expression level of genes encoding 3'-end-processing factors and the 

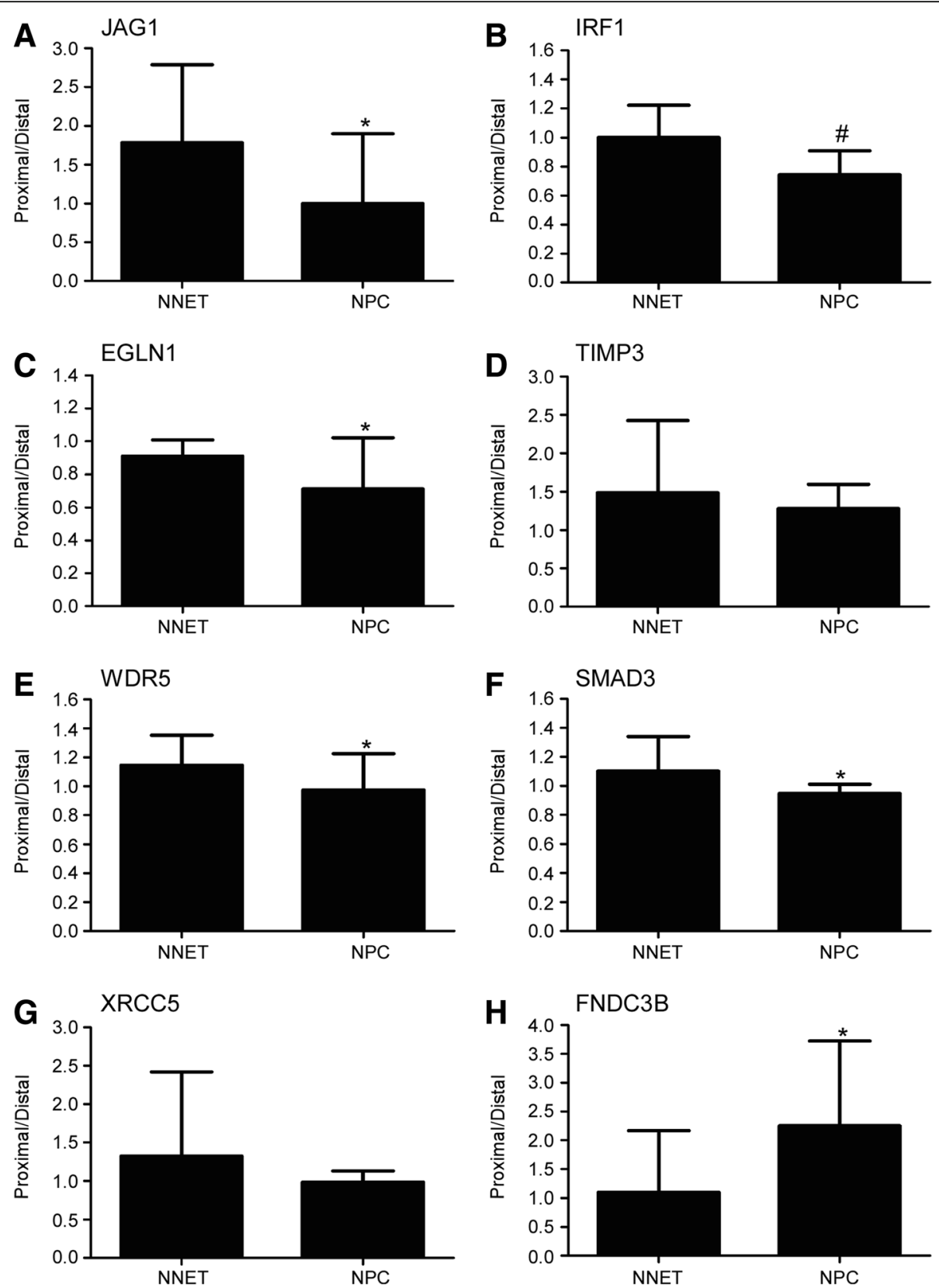

Fig. 2 Validation of 3'UTR switching in 16 NPC and NNET samples with quantitative RT-PCR. a JAG1; (B) IRF1; (c) EGLN1; (d) TIMP3; (e) WDR5; (f) SMAD3; (g) XRCC5; (h) FNDC3B. Proximal/Distal indicates the expression ratio of the shortened $3^{\prime} U T R$ to the lengthened $3^{\prime} U T R$

transcription rate of polymerase II [45]. In other studies, differential selection of APA could be ascribed to the strength of different poly(A) signals (weak or strong), and the expression levels of cleavage and polyadenylation specificity factor and cleavage-stimulating factor [49-52]. Furthermore, poly(A)-binding protein nuclear 1 , a general factor of polyadenylation, could prevent the usage of proximal poly(A) sites by direct binding to non-canonical poly(A) signals [53-55]. Moreover, the different usage of APA could change the 3'UTR length and content, leading to the loss or gain of regulatory motifs, including miRNA binding sites [20]. In our study, we found that the APA-site switching events could not change the mRNA expression levels, and it could result in the gain or loss of miRNA binding sites in the 3'UTR. For the 119 genes switched to longer 3'UTRs in NPC, totally 706 miRNA targeting sites were gained; and for the 76 genes switched to shorter 3'UTRs, totally 325 miRNA targeting sites were lost. Among them, FNDC3B was identified as the direct and 
functional target of miR-143 in liver and prostate cancer $[56,57]$. In our study, we found that FNDC3B tended to use proximal APA sites and produced mRNAs with shorter 3'UTRs in NPC. Interesting, we noticed that only the longer 3'UTRs harbored the binding sites of miR-143, suggesting that shorter mRNA transcripts can escape from miR-143 regulation. In addition, Zhang et al. reported that SMAD3 was a direct and functional target of miR-23b [58]. Here, we found that SMAD3 was prone to using distal APA sites and produced mRNAs with longer 3'UTRs in NPC and using bioinformatics analysis we confirmed that only the longer 3'UTRs harbored the binding sites of miR-23b [59]. These findings provide new insights into how APA mediate the miRNA regulation of gene expression and further affect cell biological function.

\section{Conclusions}

In summary, APA site-switching of 3'UTRs are prevalent in NPC, and APA-mediated regulation of gene expression may play important roles in NPC development and progression. Several GO terms and pathways were enriched in genes that undergo APA-switching events, including regulation of cell migration, macromolecule catabolic process, protein catabolic process, proteolysis, small conjugating protein ligase activity, and ubiquitin-protein ligase activity. These findings suggest that more detailed studies targeted genes undergoing APA site-switching events may provide novel insights into clarifying the pathogenesis of NPC and contribute to the development of novel therapeutic strategies for NPC.

\section{Additional files}

Additional file 1: PCR primers used in quantitative RT-PCR for APA
switching genes (PDF $134 \mathrm{~kb}$ )

Additional file 2: The frequency distribution of the genes with lengthened $3^{\prime} U T R$ (A) and shortened $3^{\prime} U T R$ (B) in different pairs, comparing NPC and NNET. (PDF $123 \mathrm{~kb}$ )

Additional file 3: Details of the 195 genes with significant differences in 3'UTR lengths between NPC and NNET. (XLSX $96 \mathrm{~kb}$ )

Additional file 4: Enrichment of genes with shortened $3^{\prime} U T R$ isoforms involved in various GO functional categories. (PDF 144 kb)

Additional file 5: Enrichment of genes with lengthened 3'UTR isoforms involved in various GO functional categories. (PDF $63 \mathrm{~kb}$ )

Additional file 6: Details of the 275 genes with significant differences in the mRNA levels between NPC and NNET. (XLSX $94 \mathrm{~kb}$ )

Additional file 7: The effect of APA switching events on gene expression. "Blue" and "red" colors indicate up-regulated or downregulated genes; "Green" and "yellow" colors indicate genes that switched to longer or shorter 3'UTRs. (PDF $200 \mathrm{~kb}$ )

Additional file 8: The gain or loss of miRNA binding sites in the $3^{\prime} U T R$ of genes with APA-switching events. (XLSX 26 kb)

\section{Acknowledgements}

We greatly appreciated the help of Hailiang Liu (CapitalBio Genomics Co., Ltd) for next generation sequencing data analysis.

\section{Funding}

This work was supported by grants from the National Natural Science Foundation of China (81572962, 81802705); the Natural Science Funding of Shenzhen (JCYJ20160422091914681); the Natural Science Foundation of Guang Dong Province (2017A030312003, 2017A030310468); the Health \& Medical Collaborative Innovation Project of Guangzhou City, China (201803040003); the Innovation Team Development Plan of the Ministry of Education (IRT_17R110); and the Overseas Expertise Introduction Project for Discipline Innovation (B14035).

\section{Availability of data and materials}

The raw sequencing data can be accessed from the NCBI Bioproject (http:// www.ncbi.nlm.nih.gov/bioproject) under accession no. PRJNA299088. All of the data of this study has been recorded at Sun Yat-sen University Cancer Center for future reference (RDDB2018000429).

\section{Authors' contributions}

$L L T, J M$, and YFX were responsible for study design. YFX, YQL, and LLT carried out the bioinformatic analysis of the sequencing data. YFX performed the quantitative RT-PCR, analyzed the results and drafted the manuscript. NL, XRT, XW, YS, JM participated in the data analysis. QMH and XJY collected tissue samples and performed total RNA extraction. All authors have read and approved the final manuscript.

\section{Ethics approval and consent to participate}

This study was approved by the Human Ethics Approval Committee at Sun Yat-sen University Cancer Center (GZR2015-018). Written informed consent was obtained from each study subject.

\section{Consent for publication}

Not applicable.

\section{Competing interests}

The authors declare that they have no competing interest.

\section{Publisher's Note}

Springer Nature remains neutral with regard to jurisdictional claims in published maps and institutional affiliations.

\section{Author details}

${ }^{1}$ Sun Yat-sen University Cancer Center; State Key Laboratory of Oncology in South China; Collaborative Innovation Center for Cancer Medicine;

Guangdong Key Laboratory of Nasopharyngeal Carcinoma Diagnosis and Therapy, Guangzhou 510060, People's Republic of China. ${ }^{2}$ Department of Cell Biology and Genetics, Shenzhen University Health Science Center, Shenzhen 518060, People's Republic of China.

Received: 4 May 2018 Accepted: 12 October 2018

Published online: 23 October 2018

\section{References}

1. Yu MC, Yuan JM. Epidemiology of nasopharyngeal carcinoma. Semin Cancer Biol. 2002;12:421-9.

2. Feng BJ, Huang W, Shugart $Y Y$, Lee MK, Zhang F, Xia JC, et al. Genome-wide scan for familial nasopharyngeal carcinoma reveals evidence of linkage to chromosome 4. Nat Genet. 2002:31:395-9.

3. Ou SH, Zell JA, Ziogas A, Anton-Culver H. Epidemiology of nasopharyngeal carcinoma in the United States: improved survival of Chinese patients within the keratinizing squamous cell carcinoma histology. Ann Oncol. 2007;18:29-35.

4. Ma J, Mai HQ, Hong MH, Cui NJ, Lu TX, Lu LX, et al. Is the 1997 AJCC staging system for nasopharyngeal carcinoma prognostically useful for Chinese patient populations? Int J Radiat Oncol Biol Phys. 2001;50:1181-9.

5. Xiao WW, Huang SM, Han F, Wu SX, Lu LX, Lin CG, et al. Local control, survival, and late toxicities of locally advanced nasopharyngeal carcinoma treated by simultaneous modulated accelerated radiotherapy combined with cisplatin concurrent chemotherapy: long-term results of a phase 2 study. Cancer. 2011;117:1874-83.

6. Lai SZ, Li WF, Chen L, Luo W, Chen YY, Liu LZ, et al. How does intensitymodulated radiotherapy versus conventional two-dimensional radiotherapy influence the treatment results in nasopharyngeal carcinoma patients? Int J Radiat Oncol Biol Phys. 2011;80:661-8. 
7. Lung ML, Cheung AK, Ko JM, Lung HL, Cheng Y, Dai W. The interplay of host genetic factors and Epstein-Barr virus in the development of nasopharyngeal carcinoma. Chin J Cancer. 2014;33:556-68.

8. Xu FH, Xiong D, Xu YF, Cao SM, Xue WQ, Qin HD, et al. An epidemiological and molecular study of the relationship between smoking, risk of nasopharyngeal carcinoma, and Epstein-Barr virus activation. J Natl Cancer Inst. 2012;104:1396-410.

9. Young $L S$, Dawson CW. Epstein-Barr virus and nasopharyngeal carcinoma. Chin J Cancer. 2014;33:581-90.

10. Bei JX, Li Y, Jia WH, Feng BJ, Zhou G, Chen LZ, et al. A genome-wide association study of nasopharyngeal carcinoma identifies three new susceptibility loci. Nat Genet. 2010;42:599-603.

11. Jia $W H$, Pan QH, Qin HD, Xu YF, Shen GP, Chen $L$, et al. A case-control and a family-based association study revealing an association between CYP2E1 polymorphisms and nasopharyngeal carcinoma risk in Cantonese. Carcinogenesis. 2009;30:2031-6.

12. Jiang RC, Qin HD, Zeng MS, Huang W, Feng BJ, Zhang F, et al. A functional variant in the transcriptional regulatory region of gene LOC344967 cosegregates with disease phenotype in familial nasopharyngeal carcinoma. Cancer Res. 2006;66:693-700.

13. Qin HD, Shugart YY, Bei JX, Pan QH, Chen L, Feng QS, et al. Comprehensive pathway-based association study of DNA repair gene variants and the risk of nasopharyngeal carcinoma. Cancer Res. 2011;71:3000-8.

14. Cooper TA, Wan L, Dreyfuss G. RNA and disease. Cell. 2009;136:777-93.

15. Lee SK, Calin GA. Non-coding RNAs and cancer: new paradigms in oncology. Discov Med. 2011;11:245-54.

16. Nilsen TW, Graveley BR. Expansion of the eukaryotic proteome by alternative splicing. Nature. 2010;463:457-63.

17. Tian $\mathrm{B}, \mathrm{Hu}$ J, Zhang $\mathrm{H}$, Lutz CS. A large-scale analysis of mRNA polyadenylation of human and mouse genes. Nucleic Acids Res. 2005; 33:201-12.

18. Xia Z, Donehower LA, Cooper TA, Neilson JR, Wheeler DA, Wagner EJ, et al. Dynamic analyses of alternative polyadenylation from RNA-seq reveal a 3'UTR landscape across seven tumour types. Nat Commun. 2014;5:5274.

19. Zhang H, Lee JY, Tian B. Biased alternative polyadenylation in human tissues. Genome Biol. 2005;6:R100.

20. Boutet SC, Cheung TH, Quach NL, Liu L, Prescott SL, Edalati A, et al. Alternative polyadenylation mediates microRNA regulation of muscle stem cell function. Cell Stem Cell. 2012;10:327-36.

21. Ribas J, Ni X, Castanares M, Liu MM, Esopi D, Yegnasubramanian S, et al. A novel source for miR-21 expression through the alternative polyadenylation of VMP1 gene transcripts. Nucleic Acids Res. 2012;40:6821-33.

22. Sandberg R, Neilson JR, Sarma A, Sharp PA, Burge CB. Proliferating cells express mRNAs with shortened $3^{\prime}$ untranslated regions and fewer microRNA target sites. Science. 2008;320:1643-7.

23. Hilgers V, Perry MW, Hendrix D, Stark A, Levine M, Haley B. Neural-specific elongation of 3' UTRs during Drosophila development. Proc Natl Acad Sci U S A. 2011;108:15864-9.

24. Ji Z, Tian B. Reprogramming of $3^{\prime}$ untranslated regions of mRNAs by alternative polyadenylation in generation of pluripotent stem cells from different cell types. PLoS One. 2009;4:e8419.

25. Ulitsky I, Shkumatava A, Jan CH, Subtelny AO, Koppstein D, Bell GW, et al. Extensive alternative polyadenylation during zebrafish development. Genome Res. 2012;22:2054-66.

26. Elkon $R$, Drost J, van Haaften $G$, Jenal $M$, Schrier M, Oude Vrielink JA, et al. E2F mediates enhanced alternative polyadenylation in proliferation. Genome Biol. 2012;13:R59.

27. Mayr C, Bartel DP. Widespread shortening of 3'UTRs by alternative cleavage and polyadenylation activates oncogenes in cancer cells. Cell. 2009;138:673-84.

28. An JJ, Gharami K, Liao GY, Woo NH, Lau AG, Vanevski F, et al. Distinct role of long 3' UTR BDNF mRNA in spine morphology and synaptic plasticity in hippocampal neurons. Cell. 2008;134:175-87.

29. Lau AG, Irier HA, Gu J, Tian D, Ku L, Liu G, et al. Distinct 3'UTRs differentially regulate activity-dependent translation of brain-derived neurotrophic factor (BDNF). Proc Natl Acad Sci U S A. 2010;107:15945-50.

30. Fu Y, Sun Y, Li Y, Li J, Rao X, Chen C, et al. Differential genome-wide profiling of tandem 3' UTRs among human breast cancer and normal cells by high-throughput sequencing. Genome Res. 2011;21:741-7.

31. Lin Y, Li Z, Ozsolak F, Kim SW, Arango-Argoty G, Liu TT, et al. An in-depth map of polyadenylation sites in cancer. Nucleic Acids Res. 2012;40:8460-71.
32. Morris AR, Bos A, Diosdado B, Rooijers K, Elkon R, Bolijn AS, et al. Alternative cleavage and polyadenylation during colorectal cancer development. Clin Cancer Res. 2012;18:5256-66.

33. Shepard PJ, Choi EA, Lu J, Flanagan LA, Hertel KJ, Shi Y. Complex and dynamic landscape of RNA polyadenylation revealed by PAS-Seq. RNA. 2011;17:761-72.

34. Masamha CP, Xia Z, Yang J, Albrecht TR, Li M, Shyu AB, et al. CFIm25 links alternative polyadenylation to glioblastoma tumour suppression. Nature. 2014;510:412-6.

35. Lai DP, Tan S, Kang YN, Wu J, Ooi HS, Chen J, et al. Genome-wide profiling of polyadenylation sites reveals a link between selective polyadenylation and cancer metastasis. Hum Mol Genet. 2015;24:3410-7.

36. Li Y, Sun Y, Fu Y, Li M, Huang G, Zhang C, et al. Dynamic landscape of tandem 3' UTRs during zebrafish development. Genome Res. 2012;22:1899-906.

37. Tian P, Sun Y, Li Y, Liu X, Wan L, Li J, et al. A global analysis of tandem 3'UTRs in eosinophilic chronic rhinosinusitis with nasal polyps. PLoS One. 2012;: :e48997.

38. Rhead B, Karolchik D, Kuhn RM, Hinrichs AS, Zweig AS, Fujita PA, et al. The UCSC genome browser database: update 2010. Nucleic Acids Res. 2010;38: D613-9.

39. Robinson MD, McCarthy DJ, Smyth GK. edgeR: a Bioconductor package for differential expression analysis of digital gene expression data. Bioinformatics. 2010;26:139-40.

40. Huang da W, Sherman BT, Lempicki RA. Systematic and integrative analysis of large gene lists using DAVID bioinformatics resources. Nat Protoc. 2009:4:44-57.

41. Lewis BP, Burge CB, Bartel DP. Conserved seed pairing, often flanked by adenosines of human genes are microRNA targets. Cell. 2005;120:15-20.

42. Livak KJ, Schmittgen TD. Analysis of relative gene expression data using real-time quantitative PCR and the 2(-Delta Delta C(T)) method. Methods. 2001;25:402-8.

43. Langmead B, Trapnell C, Pop M, Salzberg SL. Ultrafast and memory-efficient alignment of short DNA sequences to the human genome. Genome Biol. 2009;10:R25.

44. Agresti A. Categorical data analysis, Second Edition. Hoboken, New Jersey: John Wiley \& Sons, Inc:; 2002.

45. Elkon R, Ugalde AP, Agami R. Alternative cleavage and polyadenylation: extent, regulation and function. Nat Rev Genet. 2013;14:496-506.

46. Sun $Y, F u$ Y, Li Y, Xu A. Genome-wide alternative polyadenylation in animals: insights from high-throughput technologies. J Mol Cell Biol. 2012:4:352-61.

47. Nordlund J, Kiialainen A, Karlberg O, Berglund EC, Goransson-Kultima H, Sonderkaer $\mathrm{M}$, et al. Digital gene expression profiling of primary acute lymphoblastic leukemia cells. Leukemia. 2012;26:1218-27.

48. Lutz CS. Alternative polyadenylation: a twist on mRNA 3' end formation. ACS Chem Biol. 2008:3:609-17.

49. Proudfoot NJ. Ending the message: poly(A) signals then and now. Genes Dev. 2011;25:1770-82.

50. Yang Q, Coseno M, Gilmartin GM, Doublie S. Crystal structure of a human cleavage factor $\mathrm{CFI}(\mathrm{m}) 25 / \mathrm{CFI}(\mathrm{m}) 68 /$ RNA complex provides an insight into poly(A) site recognition and RNA looping. Structure. 2011; 19:368-77.

51. Shell SA, Hesse C, Morris SM Jr, Milcarek C. Elevated levels of the 64-kDa cleavage stimulatory factor (CstF-64) in lipopolysaccharide-stimulated macrophages influence gene expression and induce alternative poly(A) site selection. J Biol Chem. 2005;280:39950-61.

52. Takagaki Y, Seipelt RL, Peterson ML, Manley JL. The polyadenylation factor CstF-64 regulates alternative processing of IgM heavy chain pre-mRNA during B cell differentiation. Cell. 1996;87:941-52.

53. de Klerk E, Venema A, Anvar SY, Goeman JJ, Hu O, Trollet C, et al. Poly(A) binding protein nuclear 1 levels affect alternative polyadenylation. Nucleic Acids Res. 2012;40:9089-101.

54. Jenal M, Elkon R, Loayza-Puch F, van Haaften G, Kuhn U, Menzies FM, et al. The poly(A)-binding protein nuclear 1 suppresses alternative cleavage and polyadenylation sites. Cell. 2012;149:538-53.

55. Simonelig M. PABPN1 shuts down alternative poly(A) sites. Cell Res. 2012;22: 1419-21.

56. Zhang $X$, Liu S, Hu T, He Y, Sun S. Up-regulated microRNA-143 transcribed by nuclear factor kappa B enhances hepatocarcinoma metastasis by repressing fibronectin expression. Hepatology. 2009;50:490-9. 
57. Fan X, Chen X, Deng W, Zhong G, Cai Q, Lin T. Up-regulated microRNA-143 in cancer stem cells differentiation promotes prostate cancer cells metastasis by modulating FNDC3B expression. BMC Cancer. 2013;13:61.

58. Zhang $X$, Yang J, Zhao J, Zhang P, Huang X. MicroRNA-23b inhibits the proliferation and migration of heat-denatured fibroblasts by targeting Smad3. PLoS One. 2015:10:e0131867.

59. Lewis BP, Burge CB, Bartel DP. Conserved seed pairing, often flanked by adenosines, indicates that thousands of human genes are microRNA targets. Cell. 2005;120:15-20.

Ready to submit your research? Choose BMC and benefit from:

- fast, convenient online submission

- thorough peer review by experienced researchers in your field

- rapid publication on acceptance

- support for research data, including large and complex data types

- gold Open Access which fosters wider collaboration and increased citations

- maximum visibility for your research: over $100 \mathrm{M}$ website views per year

At $\mathrm{BMC}$, research is always in progress.

Learn more biomedcentral.com/submissions 